\title{
Cuerpo(s), comunicación y cultura. Balance académico sobre el cuerpo y la corporalidad como objetos de estudio de la comunicación
}

\section{Marta Rizo García ${ }^{1}$}

Recibido: 13/01/2020

Aprobado por pares: $26 / 03 / 2020$
Enviado a pares: $13 / 01 / 2020$

Aceptado: 03/04/2020

DOI: $10.5294 /$ pacla.2021.24.4.3

Para citar este artículo / to reference this article / para citar este artigo

Rizo, M. (2021). Cuerpo(s), comunicación y cultura. Balance académico sobre el cuerpo y la corporalidad como objetos de estudio de la comunicación. Palabra Clave, 24(4), e2443. https://doi.org/10.5294/pacla.2021.24.4.3

\section{Resumen}

El campo de la comunicación ha privilegiado el estudio de los llamados medios de comunicación y en mucha menor medida ha atendido procesos interpersonales e intersubjetivos. Quizás por ello no son muchas las reflexiones sobre el cuerpo y la corporalidad realizadas en el campo de la comunicación. Más allá de los estudios sobre comunicación interpersonal o de las categorías referidas a la comunicación no verbal, es sin duda insuficiente la reflexión en torno a la naturaleza corporal de la comunicación y a los cuerpos, entendidos como vehículos para la comunicación o medios de comunicación de los sujetos con el mundo. Estas páginas presentan un balance general de la presencia del cuerpo como objeto de estudio de la comunicación en el ámbito académico iberoamericano, para plantear algunos retos a la investigación en comunicación -que es también investigación de la cultura-, cuyo objeto de estudio sigue siendo, en ocasiones, puesto en cuestión. Al tratarse de un ensayo teórico, en este texto se usa una metodología documental, con cierta mirada historiográfica, pues interesa presen-

$1 \varangle$ https://orcid.org/0000-0003-3066-1419. Universidad Autónoma de la Ciudad de México, México. marta.rizo@uacm.edu.mx 
tar un panorama del lugar que ha ocupado la reflexión sobre el cuerpo en la investigación sobre la comunicación. Aunque históricamente el cuerpo y la corporalidad no han sido temas muy tomados en cuenta en la investigación en comunicación, su abordaje en nuestro campo de conocimiento da lugar a temas de investigación sugerentes en la actual ecología comunicativa, dominada, en cierto modo y en ciertos contextos, por la comunicación digital. Por ello, incorporar el cuerpo y la corporalidad como ejes temáticos en la reflexión sobre la comunicación y la cultura puede, sin duda, enriquecer, en términos teóricos y empíricos, el campo académico en cuestión.

\section{Palaras clave (fuente: tesauro de la Unesco)}

Comunicación interpersonal; corporalidad; cultura; interacción social; investigación social; proceso de comunicación. 


\section{Body(ies), Communication, and Culture. Academic Assessment of the Body and Corporality as Subject Matters of Communication}

\section{Abstract}

Communication has privileged the study of the so-called media and addressed interpersonal and intersubjective processes to a much lesser extent; perhaps, this is why there are not many reflections on the body and corporality in this field. Beyond studies on interpersonal communication or the categories referring to non-verbal communication, reflection on the corporeal nature of communication and bodies - understood as vehicles for communication or means of communication of individuals with the world-is undoubtedly insufficient. These pages present a general assessment of the body as a subject matter of communication in the Ibero-American scholarly environment to pose some challenges to communication research — which is also cultural research-, whose subject matter continues to be questioned. As a theoretical essay, this paper uses a documentary method with a specific historiographic view since it is interested in providing an overview of the place that reflection on the body has occupied in communication research. Although the body and corporality have not been topics historically considered by communication research, their approach in our knowledge domain gives rise to suggestive research topics in the current communicative ecology, overshadowed somehow and in particular contexts by digital communication. Thus, incorporating the body and corporality as theme lines in the reflection on communication and culture can undoubtedly enrich the academic field concerned theoretically and empirically.

\section{Keywords (Source: Unesco Thesaurus)}

Interpersonal communication; corporality; culture; social interaction; social research; communication process. 


\section{Corpo(s), comunicação e cultura. Balanço acadêmico sobre o corpo e a corporalidade como objetos de estudo da comunicação}

\section{Resumo}

O campo da comunicação tem privilegiado o estudo dos chamados "meios de comunicação" e, em muito menor medida, tem respondido a processos interpessoais e intersubjetivos. É provável que, por isso, não haja muitas reflexões sobre o corpo e a corporalidade realizadas no campo da comunicação. Mais além dos estudos sobre comunicação interpessoal ou das categorias referidas à comunicação não verbal, é, sem dúvidas, insuficiente a reflexão sobre a natureza corporal da comunicação e sobre os corpos, entendidos como veículos para a comunicação ou meios de comunicação dos sujeitos com o mundo. Estas páginas apresentam um balanço da presença do corpo como objeto de estudo da comunicação no contexto acadêmico ibero-americano, para propor alguns desafios para a pesquisa em comunicação - que é também pesquisa da cultura —, cujo objeto de estudo continua sendo, em muitos casos, colocado em questão. Ao se tratar de um ensaio teórico, neste texto, é usada metodologia documental, com visão historiográfica, pois interessa apresentar um panorama do lugar que a reflexão sobre o corpo tem ocupado na pesquisa sobre a comunicação. Embora historicamente o corpo e a corporalidade não tenham sido temas muito considerados na pesquisa em comunicação, sua abordagem em nosso campo de conhecimento abre espaço a temas de pesquisa sugestivos na atual ecologia comunicativa, dominada, em certo modo e em determinados contextos, pela comunicação digital. Por isso, incorporar o corpo e a corporalidade como eixos temáticos na reflexão sobre a comunicação e a cultura pode enriquecer, em termos teóricos e empíricos, o campo acadêmico em questão.

\section{Palavras-chave (Fonte: tesauro da Unesco)}

Comunicação interpessoal; corporalidade; cultura; interação social; pesquisa social; processo de comunicação. 


\section{Apunte introductorio}

La reflexión sobre el cuerpo es inherente a la reflexión sobre la comunicación humana. El intercambio de información, la construcción de significados y la producción de sentidos, los vínculos establecidos por medio de procesos comunicativos, los elementos no verbales presentes en la interacción cara a cara, entre otros muchos procesos que los sujetos experimentan cotidianamente, son producto del contacto entre dos o más cuerpos. Es tan obvia la relación que establecemos con y desde nuestros cuerpos, que muchas veces esta pasa desapercibida en los trabajos académicos. Y el campo de la comunicación no es la excepción. De ahí que no sean muchas las aportaciones sobre el cuerpo realizadas en este campo de conocimiento. Más allá de lo que suele afirmarse en estudios sobre comunicación interpersonal, a partir de categorías referidas a la comunicación no verbal, es poca o, sin duda, insuficiente la reflexión en torno a la naturaleza corporal de la comunicación humana y sobre el cuerpo, entendido como vehículo para la comunicación e interacción humanas.

La relación entre cuerpo y comunicación implica, además, tomar en cuenta la cultura y aspectos vinculados con esta, tales como las identidades, las alteridades e incluso la noción general de sociedad, pues "entre todas las señas de identidad, el cuerpo es la más definitoria, la que nos señala como individuos irrepetibles e históricos" (Castañares, 2010, p. 85). Lo micro y lo macro son dos dimensiones interdependientes en el abordaje del cuerpo y la corporalidad. Así, el cuerpo dice de quien lo porta, del sujeto corpóreo, pero el cuerpo comunica también elementos macro, referidos al contexto, al entorno cultural en que el sujeto se ubica y actúa. En este sentido, el cuerpo no es solo receptor de información y vehículo de comunicación con el mundo, pues además, en sí mismo, comunica información sobre el entorno en el que el sujeto corpóreo actúa.

A partir de las ideas anteriores, es una obviedad decir que investigar la comunicación no supone únicamente aproximarnos a los llamados medios de comunicación. Aquí retomamos lo dicho hace ya algunos años por Raúl Fuentes: "en el centro de nuestro objeto de estudio, la comunicación, no están los 'mensajes' o los 'contenidos' sino las relaciones, establecidas e 
investigadas a través de sus múltiples mediaciones, entre la producción de sentido y la identidad de los sujetos sociales en las más diversas prácticas socioculturales" (2008, p. 113). Y el cuerpo puede ser considerado, sin duda, un productor de sentido: "el cuerpo logra comunicar -y también captarun 'algo' que los discursos por sí solos no logran transmitir, justo porque nos lleva hacia el terreno de lo afectivo y lo sensorial” (Huffschmid, 2013, p. 117). Tomar en cuenta la relación entre el cuerpo y los afectos, las emociones y los sentimientos, algo que se ha privilegiado en la sociología desde la sana irrupción del denominado "giro sensorial" en las ciencias sociales, daría para otro trabajo; aquí únicamente se exponen algunas miradas que dan cuenta del potencial comunicativo del cuerpo.

En las siguientes páginas se presenta una revisión general de la presencia del cuerpo como objeto de estudio a lo largo de la historia de la investigación en comunicación, concretamente en el contexto iberoamericano y poniendo énfasis en lo producido en México. Se ofrece una suerte de estado de la cuestión que permite identificar qué se ha realizado en la materia, por un lado, y qué retos plantea la incorporación del cuerpo en el campo de la comunicación, cuyo objeto de estudio sigue siendo en ocasiones puesto en cuestión, por el otro. La metodología utilizada es la investigación documental; se revisan concretamente tres fuentes académicas: las memorias de los congresos de la Asociación Latinoamericana de Investigadores de la Comunicación (Alaic), las memorias de los congresos de la Asociación Española de Investigación de la Comunicación (AE-IC) y el Catálogo de Documentación en Ciencias de la Comunicación (CC-DOC), que da cuenta de la producción académica mexicana. Una mención aparte merece la revisión de las ponencias presentadas en la mesa "Cuerpo y comunicación" del II Encuentro Latinoamericano de Investigadores/as sobre Cuerpos y Corporalidades en las Culturas, celebrado en 2015 en Bogotá, Colombia.

\section{Aportes teóricos para pensar la relación entre cuerpo, comunicación y cultura}

Antes de presentar el balance académico es pertinente exponer algunas aportaciones teóricas generales sobre el cuerpo. Para ello, se presentan en un primer momento las propuestas de los sociólogos Erving Goffman y Pierre 
Bourdieu; posteriormente se aborda la noción de cuerpo como construcción sociocultural y, en un tercer momento, la relación cuerpo-género, que consideramos central, sobre todo por los aportes que los estudios de género y el feminismo han hecho a la investigación sobre el cuerpo y, en general, a la incorporación de lo sensocorporal-afectivo en los estudios sociales.

En Erving Goffman, el ser humano, en su corporalidad, es un actor del escenario teatral que es la realidad de la vida cotidiana. El cuerpo porta sentido, provoca significaciones y, por sí mismo, significa. El cuerpo habla y habita la realidad. Nos presentamos ante otros por medio del cuerpo, de modo que las apariencias externas, con todos sus componentes, adquieren una importancia cabal, por el significado social que les ha sido atribuido. Esta presentación ante los demás se da por medio de señales sobre todo no verbales, lo que Goffman denomina "glosario del cuerpo". Esta glosa corporal supone el "proceso mediante el cual una persona utiliza claramente los gestos corporales para que se puedan deducir otros aspectos, no apreciables de otro modo de su situación" (1979, p. 30). Las glosas corporales, por tanto, fungen como facilitadoras de las interpretaciones que cada sujeto en interacción quiere proyectar al otro. El cuerpo proyecta una imagen externa para poder vivir en sociedad. El cuerpo es usado, tiene una función, es performativo, no existe fuera de las prácticas que lo hacen posible. Como manifestaciones o expresiones de las culturas, las conductas corporales se estandarizan y generan obligatoriedad y asimilación.

Esta última idea permite introducir los aportes de Bourdieu. Para el sociólogo francés, los procesos de socialización -y, en un sentido general, la cultura- moldean el cuerpo, lo disponen a actuar de una u otra forma. Algo que pareciera individual y subjetivo, el cuerpo, aparece en la propuesta de Pierre Bourdieu como entretejido con lógicas sociales más complejas y estructurales. Las formas de percibir, sentir, hacer y pensar (lo que el autor denomina habitus) son in-corporadas, hechas cuerpo por y en los sujetos, dependiendo de sus condiciones sociales e individuales. Pensar el cuerpo desde Bourdieu implica considerar el cuerpo como "algo más que un objeto separado de una conciencia que piensa”, para pensarlo, en cambio, como "productor de un tipo particular de Sentido, un Sentido práctico" 
(Sabido, 2013, p. 39). Mientras que la propuesta goffmaniana aborda cuerpos en interacción que proyectan y reciben significados, Bourdieu concibe los cuerpos no tanto como receptáculos de significados, sino mayormente como productores de sentido por sí mismos. Nuestros hábitos corporales, por tanto, no son individuales, sino que están siempre relacionados con las sociedades en las que vivimos. Estos actos operan, entonces, en el marco de una razón práctica que los orienta. El cuerpo produce un sentido práctico que hace posible que lo que hacemos con él dé cuenta de la sociedad en la que vivimos, de sus jerarquías, diferencias y categorizaciones. En palabras del autor:

La relación con el mundo es una relación de presencia en el mundo, de estar en el mundo, en el sentido de pertenecer al mundo, de estar poseído por él, en la que ni el agente ni el objeto se plantean como tales. El grado en el que se invierte el cuerpo en esta relación es, sin duda, uno de los determinantes principales del interés y la atención que se implican en él, y de la importancia -mensurable por su duración, su intensidad, etcétera- de las modificaciones corporales resultantes [...]. Aprendemos por el cuerpo. El orden social se inscribe en los cuerpos a través de esta confrontación permanente, más o menos dramática, pero que siempre otorga un lugar destacado a la afectividad y, más precisamente, a las transacciones afectivas con el entorno social. (Bourdieu, 1999, p. 186)

En definitiva, además de tener una naturaleza biológica, el cuerpo es social y simbólico, e implica la incorporación de habitus, hexis corporales y capitales simbólicos que reproducen la lógica social. De ahí que sea válido afirmar que, desde Bourdieu, "el mundo es leído con el cuerpo". El cuerpo dispone y dice del sujeto que lo porta y, más aún, de la estructura social y cultural en la que este se halla inmerso.

\section{El cuerpo como construcción sociocultural}

Hablar del cuerpo como una construcción social y cultural remite, sin duda, a las aportaciones del antropólogo David Le Breton, que concibe el cuerpo como un dispositivo que logra explicar muchos aspectos de las culturas y las sociedades contemporáneas. El cuerpo "está en el corazón de la acción individual y colectiva, en el corazón del simbolismo social" (2001, pp. 7-8). El cuerpo dice de la sociedad en la que habita, y el cómo es concebi- 
do el cuerpo en una sociedad es indicador de los modos en que se concibe a la persona y la identidad: "las representaciones del cuerpo nos hablan de las construcciones culturales y la vinculación social de la persona y el cuerpo, nos revelan cosmologías y la forma en que una sociedad concibe qué es una persona" (p. 13).

Aunque pareciera que el cuerpo es visto como representación de la cultura y la sociedad en la que se encuentra, "no podemos separar por un lado sus representaciones y por otro cómo es vivido el cuerpo, no pueden ser separadas como si fueran distintas perspectivas, o como si fueran antagonistas" (Aguilar, 2004, p. 51). Lo individual y lo colectivo son aspectos indisolubles, y lo biológico y lo sociocultural se vinculan de forma absoluta cuando se habla de los cuerpos.

En un sentido similar a la propuesta de Le Breton, Mary Douglas (1970) habla del cuerpo como una metáfora fundamental, de orden político y social, a partir de la cual se puede entender la cultura. Para Douglas, el cuerpo es un sistema de clasificación, un medio a través del cual se representan los conceptos de orden y desorden social. Por su parte, Marcel Mauss (1991) habla de las técnicas del cuerpo para referirse a las maneras o modos en los que los seres humanos saben cómo usar sus cuerpos en la sociedad que habitan. Estas técnicas se convierten en un importante medio para la socialización de los individuos en los contextos culturales que habitan.

Es tal la relevancia del cuerpo para comprender la cultura y la sociedad que parece extraño que las ciencias sociales lo hayan tenido olvidado durante tanto tiempo. Hace unas décadas el cuerpo era tratado como un fenómeno biológico y natural, y no social y cultural; por consiguiente, no era concebido como un objeto de estudio propio de la investigación social. Afortunadamente, desde el último cuarto del siglo XX el cuerpo ha ido adquiriendo legitimidad en la sociología, la antropología y la historia, tres de las disciplinas que más lo han abordado.

Somos cuerpo. Vemos y vivimos el mundo a través de nuestro cuerpo. Nuestro cuerpo es lo primero que nos hace conscientes de nuestro ser y estar en el mundo. El cuerpo ocupa un lugar privilegiado en el proceso 
de construcción tanto de la persona como de la sociedad: "frente a la ambigüedad y la indefinición el cuerpo de los sujetos es su 'verdad' palpable, la única certeza en momentos inciertos” (Muñiz y List, 2007, p. 7).

\section{Cuerpo y género}

Es importante exponer la relación cuerpo-género, pues, como se verá posteriormente, muchos de los trabajos e investigaciones -tanto teóricas como empíricas- que abordan el tema del cuerpo tienen en el género una de sus categorías centrales. Para Judith Butler, el género "no debe interpretarse como una identidad estable o un lugar donde se asiente la capacidad de acción y desde donde resulten diversos actos, sino más bien como una identidad débilmente constituida en el tiempo, instituida en un espacio exterior mediante una repetición estilizada de actos" (2001, p. 184). De acuerdo con esta idea, el género es una construcción; no es, por tanto, algo inamovible ni permanente. Está en constante cambio, pese a que socialmente responda a estereotipos que pretenden anquilosar esta categoría en modos de ser y hacer definidos - prácticamente impuestos-, de los que poco se duda.

La cuestión del género es vital para el abordaje del cuerpo. De hecho, muchas feministas cuestionan los aportes de Foucault sobre el cuerpo, precisamente por no incorporar la cuestión del género, "que es vital para cualquier explicación del cuerpo y sobre cómo éste se ve manipulado por el poder" (Martínez, 2004, p. 133). Por su parte, para McNay no solo el género es la diferencia más fundamental entre los cuerpos, sino que el poder no es equitativo con los cuerpos femeninos y los masculinos: la dominación patriarcal del cuerpo de la mujer (1992, p. 133). Se puede decir que existen experiencias corporales diferenciadas entre hombres y mujeres.

"El cuerpo es social, socializado y socializable, tanto como lo social es corporal, corporalizado y corporalizable" (Varela, 2009, p. 97). El género es una de las dimensiones de lo social que cobra mayor importancia cuando se habla del cuerpo. Las personas viven su cuerpo de forma generizada, siempre. El hecho de que el cuerpo no haya sido un objeto de estudio primordial en las ciencias sociales se relaciona sin lugar a dudas con una visión androcéntrica dominante en la sociedad y la academia. Según Fabbri y Figueroa, la exclusión del cuerpo y de las emociones -y todo lo sensorial 
y afectivo, indefectiblemente vinculado a la experiencia corporal- como temas de interés en el campo de las ciencias sociales, "está estructurada a partir de eso que denominamos punto de vista androcéntrico, por el cual el varón y lo masculino son considerados como lo correcto, lo justo, lo apropiado, y la mujer y lo femenino como lo incompleto, lo carente, lo desviado" (2011, p. 4). Por ende, lo masculino se vuelve el punto de vista universal.

Los estudios sobre el cuerpo pueden de algún modo cuestionar esta visión androcéntrica y generar conocimiento sobre cómo las mujeres hablan de sus cuerpos, cómo se representan los cuerpos de las mujeres en los medios, de qué forma se da la violencia sobre el cuerpo de las mujeres, entre otros temas que, como veremos, afortunadamente ya están siendo atendidos en el campo de la investigación de la comunicación.

Como afirma Julieta Vartabedian (2007, p. 3) "el papel del feminismo y de algunos estudios antropológicos pueden explicar el inicial rechazo dentro de las ciencias sociales hacia la consideración exclusiva de un cuerpo que, en el caso de la mujer, es sinónimo de subordinación". Las teóricas del "feminismo de la diferencia" empezaron a hablar no solo sobre el cuerpo, sino sobre todo desde el cuerpo, para de alguna manera reivindicar de forma positiva la condición de la mujer como "lo otro".

Simone de Beauvoir es la autora que proclamó la idea que tanto se ha recuperado en los estudios de género y los estudios feministas: "no se nace mujer sino que se deviene mujer". En su obra clásica El segundo sexo (1949) hace aportaciones aún válidas para comprender que la subordinación y la opresión que viven las mujeres no son debidas a factores biológicos, sino culturales. Como afirma Vartabedian, "es el sistema patriarcal quien colocó a las mujeres en el lugar del 'segundo sexo' o la 'otra' del hombre para perpetuar el dominio masculino" (2007, p. 4).

Muchos de los estudios pioneros que relacionaron cuerpo y género permitieron desnaturalizar las identidades de género y consolidar la idea de que nociones como "hombre" y "mujer" son construcciones culturales. Fue en los años setenta cuando las feministas matizaron los conceptos de 
género y sexo para tratarlos de forma sociológica y no médica-biológica, como había pasado hasta entonces. Para el feminismo, "la dicotomía sexo/ género se convirtió en una lucha política que repudiaba la subordinación de la mujer. El 'sexo' estaba determinado por factores biológicos, el 'género' por sociales y culturales” (Vartabedian, 2007, p. 4).

Desde los años ochenta, esta dicotomía ha sido criticada por teóricas y teóricos que consideran que lo biológico (lo asociado al sexo) es también una construcción cultural. Aquí vale la pena hacer mención de feministas deconstructivistas como Judith Butler (2001), que ya citamos, o Donna Haraway (1995), quienes en sus propuestas buscan erradicar estas dicotomías y distinciones, tan ancladas socialmente. Muchas de estas críticas van acompañadas "de una clara tendencia a la descorporeización de las identidades. Para precisar aún más, se pretende abandonar la idea de un cuerpo sexuado que determina la forma de actuar de los géneros” (Vartabedian, 2007, p. 6).

Desde los años noventa, la superación del dualismo cartesiano mente/cuerpo parece no ponerse en duda, si bien tampoco puede hablarse de un determinismo absoluto de lo social sobre el cuerpo, cuya materialidad no puede ni debe negarse.

\section{El cuerpo en la investigación académica iberoamericana en comunicación: balance crítico}

Realizar un balance académico de la relación entre cuerpo y comunicación es algo complicado en un campo como el de la comunicación, que se ha caracterizado por privilegiar el estudio de los medios por sobre cualquier otro fenómeno comunicativo. No obstante, y aunque pueda parecer paradójico, la propia naturaleza interdisciplinaria del fenómeno comunicativo permite lecturas sociológicas, filosóficas, antropológicas, etc., que en algunos casos toman al cuerpo como objeto de reflexión, fundamentalmente teórica y, en mucha menor medida, resultado de análisis empíricos.

El ejercicio que presentamos en estas páginas es únicamente un apunte en torno a la presencia del cuerpo en los estudios sobre comunicación en 
el campo académico iberoamericano, con énfasis en el contexto mexicano. Para ello, realizamos una búsqueda en las memorias de los congresos del 2016 y 2018 de la Asociación Latinoamericana de Investigadores de la Comunicación (Alaic) y de algunas de las últimas ediciones de los congresos de la Asociación Española de Investigadores de la Comunicación (AE-IC), por un lado, y en el Catálogo de Documentación en Ciencias de la Comunicación, que da cuenta de la producción académica mexicana, por el otro. Cabe mencionar que en ningún momento se busca que esta muestra documental sea estadísticamente representativa, puesto que únicamente queremos aportar algunos elementos clave que nos permitan indicar algunos de los caminos por donde transcurre la reflexión sobre la comunicación y el cuerpo en nuestro campo de conocimiento. Para completar el estado de la cuestión, nos fijamos también en la presencia de abordajes comunicativos sobre el cuerpo en las ponencias presentadas en dos de las tres ediciones del Encuentro Latinoamericano de Investigadores/as sobre Cuerpos y Corporalidades en las Culturas, celebradas en 2012 y 2015 en Argentina y Colombia. Omitimos los trabajos presentados en la edición de 2018 en México porque aún no se encuentran publicadas las memorias.

\section{La investigación sobre cuerpo y comunicación en América Latina y España}

En un primer momento, se presenta el resultado de la sistematización de las ponencias presentadas en congreso de la Alaic, celebrado en octubre de 2016 en México. De todas las ponencias presentadas, se toman en cuenta las 16 que tienen al cuerpo como uno de los ejes básicos, teóricos y/o empíricos. Estos 16 trabajos se encuentran en cuatro grupos de investigación distintos, lo cual ya nos habla de la dispersión del objeto de estudio "cuerpo" o, al menos, de su carácter transversal en la investigación en comunicación. En concreto, encontramos ponencias sobre el cuerpo en los siguientes grupos: Discurso y Comunicación (3), Comunicación y Estudios Socioculturales (9), Comunicación Intercultural y Folkcomunicación (3) y Comunicación y Decolonialidad (1). En términos generales, predominan trabajos empíricos sobre dos ejes temáticos básicos: el género y la sexualidad, y destaca la presencia de ponentes de Colombia, Brasil, Argentina, México y Perú. 
En el grupo Discurso y Comunicación se presentaron dos trabajos sobre cine, uno de ellos enfocado en la manera como las audiencias infantiles interpretan los estereotipos de género de ciertos personajes del cine de Walt Disney, y un trabajo teórico cuyo eje de reflexión es la concepción del cuerpo como un acontecimiento semiótico. Las nueve ponencias del grupo de Comunicación y Estudios Socioculturales, en las que se repiten las referencias a autores como Foucault, Giddens y Butler, representan más de la mitad de todos los trabajos referidos en este punto. De los nueve trabajos, tres son teóricos y se basan en temas como el performance, el cuerpo como operador simbólico en la industria de la moda y la vivencia del dolor según las aproximaciones de autores como Le Breton. El resto, los seis trabajos empíricos, giran en torno a dos temas básicos: el de las sexualidades periféricas, presente en trabajos sobre las representaciones de la comunidad LGBTI en contextos educativos, las vivencias corporales y de género en la comunidad homosexual bear o las nuevas formas de masculinidad a partir de las visiones de lo queer propuesto por Judith Butler (2002); el segundo tema es el de las mujeres, que aparecen en trabajos sobre el cuerpo de la mujer en la prensa colombiana, la construcción estereotipada de los cuerpos de las mujeres que participan en los noticieros televisivos en Colombia o la relación entre cuerpo, mujer y violencia de género en mujeres raperas mexicanas, entre otros temas. De los tres trabajos presentados en el grupo sobre Comunicación Intercultural y Folkcomunicación, dos son teóricos y abordan respectivamente la relación cuerpo-alteridad y una ontología de la comunicación a partir de la autonomía del pensamiento-cuerpo, y el tercero da cuenta de una investigación empírica sobre el papel del cuerpo en la vivencia de lo religioso. Por último, la ponencia del grupo Comunicación y Decolonialidad es un texto teórico sobre la representación del cuerpo de las mujeres subalternizadas en los medios, desde el enfoque del feminismo decolonial.

Con respecto al congreso del año 2018, existen 23 trabajos que reflexionan en torno al cuerpo desde distintos puntos de vista y con distintos énfasis. Estos trabajos están divididos en ocho grupos de trabajo y un grupo de investigación, aún no consolidado como grupo de trabajo. Con respecto a los grupos de trabajo, la distribución fue la siguiente: Comunicación Inter- 
cultural y Folkcomunicación (1); Comunicación y Educación (1); Teoría y Metodología de la Investigación en Comunicación (2); Comunicación y Estudios Socioculturales (2); Comunicación Publicitaria (2); Discurso y Comunicación (2); Comunicación y Ciudad (1) y Comunicación Digital (1). Curiosamente, fue en el Grupo de Investigación (aun no consolidado como grupo de trabajo) donde se presentaron un mayor número de trabajos referidos al cuerpo y la corporalidad. Nos referimos al grupo dedicado a Comunicación, Género y Diversidad Sexual, que concentró 11 ponencias sobre el tema, la mayoría vinculadas con el cuerpo de la mujer y la violencia de género, tanto en productos mediáticos como en espacios públicos. Como vimos en un apartado anterior de este texto, la relación cuerpo-género es crucial en los estudios sobre el cuerpo, al menos en el ámbito de la investigación en comunicación latinoamericana.

Concluimos el panorama sobre la presencia del cuerpo en la investigación en comunicación en América Latina con la referencia a las ponencias presentadas en dos ediciones del Encuentro Latinoamericano de Investigadores/as sobre Cuerpos y Corporalidades en las Culturas. El primer encuentro se celebró en Rosario, Argentina, en 2012. En dicho congreso se plantearon un total de 16 grupos de trabajo, ninguno de ellos dedicado explícitamente a la relación cuerpo-comunicación. No obstante, es posible encontrar algunas reflexiones relacionadas con la comunicación en varios de los trabajos. En total, detectamos siete trabajos distribuidos en tres mesas distintas que, de forma más o menos directa, vinculan el cuerpo con la comunicación, en un sentido mediático o en un sentido estético-literario.

En el segundo encuentro, celebrado en Bogotá en octubre de 2015, sí hubo una mesa dedicada a Cuerpo y Comunicación. De las 12 ponencias presentadas, tres fueron teóricas y nueve empíricas. En cuanto a las teóricas, vemos que existe consenso en considerar al cuerpo como productor y receptor de significados, y los autores más mencionados para tales reflexiones son Goffman, Bourdieu y Merleau-Ponty; vemos, además, que existe una presencia más o menos equilibrada de abordajes sociológicos y filosóficos en la reflexión teórica sobre el cuerpo como ente comunicante; una tercera consideración es que los tres trabajos teóricos ponen énfasis en la 
necesidad de romper con la dicotomía mente-cuerpo; y, por último, destaca también la presencia en dos trabajos del abordaje foucaultiano sobre el cuerpo, a partir de la noción de cuerpos dóciles que propuso el autor. Con respecto a las ponencias que dan cuenta de investigaciones empíricas, vemos que predominan los análisis de las representaciones del cuerpo en medios como la fotografía, el cine, la prensa y la publicidad de alimentos; en segundo lugar, destaca la presencia de trabajos sobre la relación entre cuerpo, género y sexualidad, que abogan por la ruptura del esquema genérico binario y de la visión heteronormativa que existe sobre las vivencias de los cuerpos sexuados; por último, vemos también abordajes empíricos que relacionan cuerpo y comunicación en manifestaciones artísticas específicas como la danza y el baile.

Con respecto a la presencia del cuerpo en la investigación en comunicación en el Estado español, optamos por revisar los trabajos presentados en los congresos de la Asociación Española de Investigación de la Comunicación (AE-IC). Por el volumen de trabajos, haremos referencia únicamente a los congresos celebrados en 2008, 2010, 2016 y 2018, el último. Cabe mencionar como dato general que son realmente pocos los trabajos que tienen al cuerpo y la corporalidad como ejes de reflexión centrales. Más bien hemos encontrado referencias secundarias al cuerpo. Y nuevamente, se observa que el género y la sexualidad son los dos principales temas en torno a los cuales aparecen reflexiones sobre el cuerpo y la comunicación.

En 2008 se presentaron tres trabajos empíricos en el grupo de Estudios del Discurso: una ponencia sobre el culto al cuerpo en programas televisivos de no ficción, otra sobre el cuerpo en la ficción televisiva -a partir de la noción de ritual de Erving Goffman- y otra sobre el cuerpo en los dibujos animados y los programas de ficción para públicos infantiles. En el congreso de 2010 hubo cuatro ponencias: una teórica y tres empíricas; la teórica aborda la relación cuerpo-alma en el debate epistemológico sobre la comunicación, mientras que las empíricas abordan los cuerpos andróginos en la publicidad televisiva (trabajo presentado en el grupo de Estudios del Discurso), así como el culto al cuerpo y los trastornos alimentarios (trabajos presentados en el grupo de Estudios de Audiencia y Recepción). En el 
congreso de 2016 se presentaron únicamente dos ponencias que abordan el cuerpo: la primera se presentó en el grupo de Género y Comunicación y tuvo como eje las representaciones de género en las redes de contacto, a partir de las ideas de la heteronormatividad y los rituales de Goffman; la segunda fue expuesta en el grupo de Estudios sobre el Discurso y versó sobre el cuerpo y la violencia en la prensa de nota roja en México. Por último, en el congreso celebrado en 2018, encontramos un total de seis trabajos referidos al cuerpo: uno en el grupo de Estudios de Audiencia y Recepción, tres ponencias en el grupo de Estudios de Género y Comunicación, un trabajo en la sección temática de Historia de la Investigación en Comunicación y una intervención en la plenaria dedicada a Psicología de los Medios y Conocimiento.

Con el fin de ofrecer un balance escueto de lo hallado en torno a la presencia del cuerpo en la investigación en comunicación en América Latina y el Estado español, se mencionan a continuación algunas consideraciones generales:

1. Predominan los trabajos empíricos, en detrimento de la reflexión teórica.

2. Los autores que más se repiten como referentes teórico-conceptuales de los trabajos son Foucault, Goffman, Butler y Le Bretón.

3. Muchos de los trabajos empíricos refieren, sobre todo en el campo español, a las representaciones del cuerpo en los medios, especialmente en la televisión (de ficción y no ficción, con gran presencia de trabajos que abordan el discurso publicitario) y la publicidad.

4. El género y la sexualidad son dos ejes temáticos básicos tanto en América Latina como en el Estado español.

5. Aspectos como la moda, la religión, la música y otras manifestaciones artísticas están más abordados en el campo académico latinoamericano, con lo cual podemos afirmar que en la investigación española en comunicación parece que aún se asocia investigar la comunicación con investigar los medios. 
6. La idea anterior se plasma en el predominio de trabajos presentados en el grupo de trabajo Estudios del Discurso, en el caso de la AE-IC, mientras que en Latinoamerica vemos que la mayoría de ponencias se presentaron en el grupo de Comunicación y Estudios Socioculturales.

7. Las metodologías empleadas incluyen, en gran parte de los trabajos empíricos, análisis del discurso, análisis de contenido y métodos cualitativos dialógicos, tales como las entrevistas y los grupos de discusión.

\section{La investigación sobre cuerpo y comunicación en México}

Para la exploración de lo producido en México, se tomó en cuenta el Catálogo de Documentación de Ciencias de la Comunicación. La búsqueda la realizamos en enero de 2020, obteniendo un total de 48 textos, sobre los cuales presentamos algunas consideraciones a continuación. Con textos que abarcan un periodo de 25 años, de 1991 a 2018, en una primera lectura vemos que predominan los ensayos teóricos y, en menor medida, los abordajes empíricos. Cabe mencionar, además, que se aprecia una presencia bastante notoria de autores como Foucault, Goffman y Bourdieu, y también vemos que algunos de los trabajos parten de una perspectiva semiótica y usan herramientas como el análisis del discurso. Para clasificar los textos que tomamos en cuenta, determinamos cinco áreas temáticas: a) artes y estética, con 7 trabajos; b) cuerpo e identidades, con 16 trabajos, divididos a su vez en los subtemas género (13) y jóvenes (3); c) cuerpo-medios, con trabajos divididos, a su vez, en los que abordan los medios tradicionales (12) y los que se centran en los medios digitales (9); d) cuerpo-Interacción, con 2 ponencias; e) nuevas lecturas (otros enfoques), también con 2 ponencias.

Los trabajos agrupados en el área de Artes y Estética, en total 7, son en su mayoría ensayos teóricos sobre la representación del cuerpo en diferentes manifestaciones artísticas, como las artes escénicas y las visuales. Destacan el teatro, la pintura y la danza. En los ensayos se ve el cuerpo como "objeto" de análisis, como elemento estético y como dispositivo utilizado por y para el arte.

Con respecto al rubro temático Cuerpo e Identidades, se agruparon los 16 trabajos en dos subtemas: género (13) y jóvenes (3). Muchos de los 
trabajos que abordan la relación entre cuerpo, comunicación y género son artículos con tratamiento empírico, en los que prevalece la perspectiva de género. Algunos de sus temas son: la violencia sobre el cuerpo de las mujeres, la mediación de género en los imaginarios sociales sobre las relaciones amorosas y la sexualidad, la construcción de las masculinidades, el cuidado del cuerpo y su relación con la imagen corporal, entre otros. De estos trabajos destacan dos ideas básicas: primera, el enfoque de género sigue privilegiando el estudio de las mujeres muy por encima del estudio de los hombres; y segunda, el estudio del cuerpo y el género se aterriza en muchas ocasiones en abordajes empíricos sobre el amor y la sexualidad. El segundo subtema dentro del área temática Cuerpo e Identidades tiene que ver con los jóvenes. En este rubro se hallaron reflexiones teóricas y abordajes empíricos sobre el cuerpo de los jóvenes como expresión de la identidad juvenil, sobre la estética juvenil y los adornos corporales, y sobre el baile popular y el grafiti como estrategias de resistencia juvenil, a través del cuerpo, en ciudades latinoamericanas.

De los 21 trabajos que abordan la relación Cuerpo-Medios, 12 se centran en medios tradicionales y 9 en medios digitales-virtuales. Con respecto a los trabajos que vinculan cuerpo y medios tradicionales, se observan análisis de los estereotipos de los cuerpos jóvenes -vistos como objetos de consumo- en los medios de comunicación, especialmente la televisión; los estereotipos corporales vinculados a la salud y los hábitos alimentarios en programas de televisión infantil y en telenovelas mexicanas; lo grotesco en las representaciones del cuerpo de la mujer en expresiones musicales masivas; el cuerpo en diferentes expresiones cinematográficas; la presencia del cuerpo en las campañas de marketing político que construyen cierta imagen de los candidatos políticos en tiempos electorales; o la representación del cuerpo femenino en la literatura. Predominan los análisis del discurso y análisis de contenido. Con respecto a los trabajos que abordan el cuerpo en entornos mediáticos digitales, la mayoría escritos en la última década tienen una fuerte presencia la categoría "virtualidad" y la reflexión sobre lo que puede denominarse "comunicación sin cuerpo". Existen trabajos empíricos que analizan los "cuerpos virtuales" (avatares) en interacción en los espacios electrónicos o la interacción cuerpo-máquina; otros que po- 
nen énfasis en el concepto de simulación aplicado a determinados universos fílmicos; y también ensayos teóricos que presentan reflexiones sobre la descorporización del sujeto en el contexto de las rearticulaciones del espacio y el tiempo en los entornos digitales.

Los dos últimos rubros tienen que ver con la relación Cuerpo-Interacción, por un lado, y con nuevas lecturas o enfoques para el abordaje del cuerpo en relación con la comunicación y, en un sentido amplio, con la cultura. En ambos casos se encontraron 2 trabajos. En el rubro Cuerpo-Interacción, 2 ensayos teóricos: uno que resalta el papel del cuerpo en las interacciones humanas, poniendo énfasis en categorías de la comunicación interpersonal no verbal, y otro que plantea la propuesta de una semiótica del cuerpo y de los sentidos, a partir de autores como Kant y Peirce, y con conceptos como tiempo y experiencia-además de cuerpo- en el centro. Por último, como Nuevas Lecturas se hallaron 2 ensayos, también de corte teórico: el primero tiene como eje básico de reflexión el cuerpo desde el punto de vista de los estudios subalternos, mientras que el segundo se centra en el análisis del poder como efecto de procesos de construcción de sentido que involucran el cuerpo, el lugar y el discurso.

A modo de síntesis de este breve panorama de la presencia del cuerpo en los estudios de comunicación en México, pueden destacarse los siguientes elementos:

1. Predominan los estudios de corte sociocultural, muy por encima de trabajos de corte filosófico e interpretativo.

2. La noción de intersubjetividad-que nos parece especialmente útil para profundizar en los abordajes no mediáticos de la comunicaciónes prácticamente inexistente en este conjunto de trabajos que abordan la relación entre el cuerpo y la comunicación.

3. Destacan trabajos sobre el cuerpo de la mujer con el enfoque de los estudios de género. Aquí es importante mencionar que son casi nulas las referencias a la teoría feminista, pese a que, como vimos páginas atrás, el feminismo ha sido uno de los frentes académicos básicos del abordaje de la relación cuerpo-género. 
4. En las investigaciones empíricas sobre cuerpo y medios es notable el predominio de trabajos sobre las representaciones del cuerpo en medios como la televisión y el cine, a partir de estrategias metodológicas como el análisis del discurso.

\section{Cierre: retos para la investigación en comunicación}

La comunicación humana es comunicación desde el cuerpo, para el cuerpo y entre cuerpos. Somos conscientes de la existencia de los otros con quienes nos comunicamos porque tenemos conciencia perceptiva de nuestro cuerpo y porque, con base en nuestro esquema corporal, constituimos las imágenes que tenemos de los otros y somos capaces de interactuar con él. El cuerpo, por tanto, no solo recibe, sino que sobre todo crea y significa. El lenguaje solo es posible si emana de los cuerpos vivientes y sintientes, $y$ como tal es la instancia que unifica al ser. Nuestra relación con el mundo, entonces, está dada por el cuerpo; de ahí que el cuerpo pueda ser considerado como el espacio universal, pues sin cuerpos no habría espacialidad. Por medio de nuestro cuerpo accedemos al mundo y, desde ese lugar, desde nuestro esquema corporal, nos comunicamos con otros, a quienes percibimos como semejantes y, simultáneamente, como diferentes: como otros. "La sociedad es, ante todo y por encima de todo, una actividad corporal" (Collins, 2009, p. 56). Al cuerpo se le asignan sentidos y, simultáneamente, el cuerpo produce sentido, permite ver lo social, la estructura. El cuerpo, o el sujeto-cuerpo, diría Merleau-Ponty, es un cuerpo situado, es decir, construido histórica, social y culturalmente.

Es posible entender el cuerpo no solo como un "nudo de significaciones vivientes" (Esteban, 2004), sino también como un cuerpo perceptivo, que es precisamente lo que reivindica el viraje de la sociología de los sentidos a la sociología de la percepción (Sabido, 2016). Lo que no puede ponerse en duda es que "el uso que se hace del cuerpo depende de las culturas” (Noguez, 2009, p. 68).

Los cuerpos ejercen toda su potencia significativa sobre los sujetos. Es desde los cuerpos que los seres humanos significan sus entornos, dotan de 
sentido a los otros seres, al espacio y al tiempo que habitan. No podemos, por tanto, comprendernos a nosotros mismos como cuerpos si no es a partir del despliegue significativo -y comunicativo- hacia los otros, partiendo de nuestro esquema corporal. $Y$, a la vez, no podemos comprender nuestras sociedades si no es a través de lo que sobre ellas comunican nuestros cuerpos.

Ser cuerpo es ser-del-mundo. El cuerpo, entonces, es un dador de sentido: recibe significados y, simultáneamente, comunica sentidos. Sea como productor de sentidos, como reflejo de lo social, como receptáculo de significados en situaciones cara a cara o como cuerpo-sujeto pensante del que emana la conciencia de sí y de los otros, el cuerpo es nuestro principal vehículo de comunicación con el mundo. Por eso, muchas veces no se concibe como un fenómeno que pueda ser analizado, pues somos cuerpo, y desde él vemos, con él actuamos.

El campo de la comunicación no ha prestado suficiente atención al cuerpo como medio de comunicación del sujeto con el mundo. En la revisión presentada en estas páginas, se aprecia la escasez de aproximaciones de corte filosófico que, además, podrían nutrir los trabajos sobre la intersubjetividad y la comunicación. Siguen predominando, especialmente en el campo de la investigación española, estudios empíricos sobre la imagen del cuerpo en diferentes medios, con enfoque de género y énfasis en análisis de los discursos televisivos, ficcionales, no ficcionales y publicitarios. Ello se explica, en parte, porque el campo de la comunicación sigue muy centrado en los medios y deja de lado otras expresiones y fenómenos comunicativos cuyo potencial significativo es enorme. Uno de ellos es, sin lugar a dudas, el cuerpo.

Este balance no ha pretendido la exhaustividad, sino más bien ilustrar de forma general qué tanta importancia se otorga al cuerpo en la investigación de la comunicación Latinoamérica y España. Como hemos visto, son prácticamente inexistentes los ensayos teóricos que articulan cuerpo, comunicación e intersubjetividad. En este sentido, el cuerpo sigue siendo visto como objeto de análisis y no se piensa suficientemente en su potencial comunicativo per se. El campo de la comunicación intersubjetiva -uno de cuyos ejes temáticos centrales es, precisamente, el cuerpo- tiene entre 
sus retos realizar trabajos de corte empírico, que no solo ayuden a fortalecer la reflexión sobre la puesta en escena de la intersubjetividad, sino que, además, ofrezcan datos sobre las diferentes manifestaciones del cuerpo y la corporalidad en situaciones de comunicación concretas, debidamente contextualizadas en una cultura y una sociedad específicas.

En definitiva, ¿̇por qué es importante que el campo de la comunicación tome en cuenta el cuerpo como tema de estudio? En primer lugar, porque el cuerpo es, como ya vimos, uno de los principales dispositivos de construcción de sentido con el que cuentan los seres humanos y, como tal, es el principal vehículo para la comunicación humana. En segundo lugar, porque, como da cuenta el balance académico realizado, muchos estudios sobre el cuerpo desde la comunicación ponen énfasis en el género y, especialmente, en las mujeres, y ello permite hablar de una línea de investigación que puede consolidarse cada vez más: cuerpo, comunicación y género, tres elementos que parece indispensable que tome en cuenta la investigación de la comunicación -más allá de los medios-. Y, por último, es importante que el campo de la comunicación siga abriendo el abanico de objetos de estudio posibles que lo alejen de la concepción tradicional de la comunicación, comúnmente asociada a los medios y a la transmisión de información por canales o vehículos técnicos. Si la investigación de la comunicación quiere seguir expandiéndose y dando cuenta del potencial que tiene para explicar las sociedades actuales, es de vital importancia que atienda problemáticas y áreas de estudio no necesariamente vinculadas con la comunicación mediada.

El cuerpo, las emociones y, en general, la dimensión sensoafectiva del mundo conforman un espacio temático sugerente para que el campo de la comunicación ofrezca más y mejores explicaciones sobre las personas en el mundo actual.

\section{Referencias}

Aguilar-Ros, A. (2004). Social Anthropology. Manchester: University of Manchester. 
Bourdieu, P. (1999). Meditaciones pascalianas. Barcelona: Anagrama.

Butler, J. (2001). El género en disputa. El feminismo y la subversión de la identidad [1990]. México: Paidós, UNAM.

Butler, J. (2002). Cuerpos que importan. Sobre los límites materiales y discursivos del 'sexo'. Buenos Aires: Paidós.

Castañares, W. (2010). El uso de la fotografía en la autorrepresentación de los sujetos en las redes sociales. En Torregrosa, M. (coord.). Imaginar la realidad. Ensayos sobre la representación de la realidad en el cine, la televisión y los nuevos medios (pp. 69-90). Sevilla-Zamora: Comunicación Social.

Collins, R. (2009). Cadenas de rituales de interacción. Barcelona: Anthropos.

Douglas, M. (1970). Natural symbols: Explorations in cosmology. Londres: Vintage.

Esteban, M. L. (2004). Antropología del cuerpo. Género, itinerarios corporales, identidad y cambio. Barcelona: Bellaterra.

Fabbri, L. y Figueroa, N. (2011). ¿Ocultos y dominados? El (no) lugar de los cuerpos en la teoría política androcéntrica. $\mathrm{X}$ Congreso de la Sociedad Argentina de Análisis Político (SAAP), Córdoba.

Fuentes Navarro, R. (2008). La construcción de la comunidad académica de la comunicación en México y Latinoamérica (2000). En La comunicación desde una perspectiva sociocultural. Acercamientos y provocaciones 1997-2007 (pp. 101-114). Guadalajara: Iteso.

Goffman, E. (1979). Relaciones en público. Microestudios del orden público. Madrid: Alianza.

Haraway, D. (1995). Ciencia, cyborgs y mujeres. La reinvención de la naturaleza. Madrid: Cátedra. 
Huffschmid, A. (2013). La otra materialidad: cuerpos y memoria en la vía pública”. En Aguilar, M. Á., Soto Villagrán, P. (coords.), Cuerpos, espacios y emociones. Aproximaciones desde las ciencias sociales (pp. 111-138). México: Porrúa, Universidad Autónoma Metropolitana Iztapalapa.

Le Breton, D. (2001). Sociología del cuerpo [1992]. Buenos Aires: Nueva Visión.

Martínez Barreiro, A. (2004). La construcción social del cuerpo en las sociedades contemporáneas. Papers, 73, 127-152. DOI: https://doi. org/10.5565/rev/papers/v73n0.1111

Mauss, M. (1991). Técnicas y movimientos corporales. En Sociología y antropología [1934] (pp. 337-356). Madrid: Tecnos.

McNay, L. (1992). Foucault and feminism: Power, gender and the self. Cambridge: Polity Press.

Muñiz, E. y List, M. (coords.) (2007). Pensar el cuerpo. México: UAM Azcapotzalco.

Noguez, E. E. (2009). Narrativas sobre la experiencia de la transformación corporal estética en una mujer. Medellín: Universidad de San Buenaventura, Facultad de Psicología.

Sabido Ramos, O. (2013). Los retos del cuerpo en la investigación sociológica. Una reflexión teórico-metodológica. En Aguilar, M. Á. y Soto Villagrán, P. (coords.), Cuerpos, espacios y emociones. Aproximaciones desde las ciencias sociales (pp. 19-54). México: Porrúa, Universidad Autónoma Metropolitana Iztapalapa. DOI: https:// doi.org/10.1016/j.df.2016.04.002

Sabido Ramos, O. (2016). Cuerpo y sentidos: el análisis sociológico de la percepción. Debate Feminista, 51, 63-80. 
Varela, S. (2009). Habitus: una reflexión fotográfica de lo corporal en Pierre Bourdieu. Iberfórum, 4(7), 94-107.

Vartabedian, J. (2007). El cuerpo como espejo de las construcciones de género. Una aproximación a la transexualidad femenina. Quaderns-e, 10. Recuperado de: https://www.raco.cat/index.php/ QuadernseICA/article/view/109038/136554 